\title{
Detection of Brain Tumor by Medical Image Processing
}

\author{
Shivangi Mahajan*, Sakshi Saini \\ Banasthali Vidyapith, Jaipur, Rajasthan (India)
}

\begin{abstract}
Medical image processing is the most inspiring and developing field today. This paper labels the method of discovery \& removal of brain tumor from patient's MRI scan images of the brain. In this paper, a technique for separation of brain tumor has been developed on 2D-MRI facts which allow the documentation of tumor tissue with great accuracy and reproducibility compared to manual techniques. The first step of discovery of brain tumor is to patterned the symmetric and asymmetric Form of brain which will define the irregularity After this step the next step is segmentation which is built on two techniques 1) F-Transform (Fuzzy Transform) 2) Morphological operation. These two techniques are used to project the image in MRI. Now by this help of project we can sense the boundaries of brain tumor and calculate the real area of tumor.
\end{abstract}

Keywords: Brain tumor, medical image processing, MRI.

Article Info: Received 18 June 2019; $\quad$ Review Completed 28 July 2019; $\quad$ Accepted 03 Aug 2019; Available online 18 August 2019

Cite this article as:

Mahajan S, Saini S, Detection of Brain Tumor by Medical Image Processing, Journal of Drug Delivery and Therapeutics. 2019; 9(4-s):709-713 http://dx.doi.org/10.22270/jddt.v9i4-s.3376

*Address for Correspondence:

Shivangi Mahajan, Banasthali Vidyapith, Jaipur, Rajasthan (India)

\section{Introduction:}

Tumor is defined as the irregular growth of the tissues (1). Epilepsy is a brain malady in which bunches of nerve cells, or neurons, in the brain occasionally signal abnormally(2) Neurons usually generate electrochemical instincts that act on other neurons, glands, and muscles to crop human thoughts, feelings, and actions(3). In epilepsy, the usual pattern of neuronal activity converts disturbed, causing strange sensations, emotions, and behaviour or sometimes convulsions, muscle spasms, and harm of consciousness(4).Medical imaging technique is used to craft visual image of interior of the human body for medical determination and non-ivassive options can be analyzed by this technology .

The brain tumor is of two stages:-

1) Primary stage.

2) Secondary stage.

When tumor range in any share of brain then it is known as brain tumor. Now when brain tumor can known number of symptoms including seizures, mood changing, difficulty in walking and hearing, vision, and muscular drive etc. brain tumor is underground into Gliomas, medullo blastoma, epeldymomas, CNS lymphoma and oligodendrogloma.

Brain tumors affect the humans badly, because of the abnormal growth of cells within the brain. It can disrupt proper brain function and be life-threatening. Two types of brain tumors have been identified as Benign tumors and Malignant tumors. Benign tumors are less harmful than malignant tumors as malignant are fast developing and harmful while benign are slow growing and less harmful. Brain tumors affect the humans badly, because of the abnormal growth of cells within the brain. It can disrupt proper brain function and be life-threatening. Two types of brain tumors have been identified as Benign tumors and Malignant tumors. Benign tumors are less harmful than malignant tumors as malignant are fast developing and harmful while benign are slow growing and less harmful.

Brain tumors affect the humans badly, because of the abnormal growth of cells within the brain. It can disrupt proper brain function and be life-threatening. Two types of brain tumors have been identified as Benign tumors and Malignant tumors. Benign tumors are less harmful than malignant tumors as malignant are fast developing and harmful while benign are slow growing and less harmful

\section{Brain tumor:}

Brain tumor is one of the most common main sources for the growth in Humanity among families and grownups in the world. Brain tumor is a group of irregular cells that produces inside of the intelligence or everywhere the brain. Various changed types of brain tumors occur. Some brain tumors are 
noncancerous (benevolent), and some brain tumors are cancerous (malicious).Early discovery of the brain tumor is very vital and the inspiration for further educations. In the brain magnetic resonance imaging (MRI), the tumor may appear clearly but for further treatment, the physician also needs the quantification of the growth area. With a occurrence of less than $1 \mathrm{~h}$ in the cowboy movie populace, brain tumors are not very common, however they are among the most fatal tumors (DeAngelis (2001))(5).The word "tumor" is of latin source and resources enlargement. Today, it is repeatedly associated with a neoplasm, which is caused by unrestrained cell propagation.(6)Brain tumors can be categorized according to their basis or degree of fierceness. Primary brain growths arise in the brain, while metastatic brain tumors regularly originate from further chunks of the group.

The term tumor is a synonym for a word neoplasm which is formed by an irregular development of cells Tumor is somewhat totally dissimilar from tumor.

\section{Types of Tumor:}

Three common types of brain tumor:

1) Benign;

2) Pre-Malignant;

3)Malignant (cancer can only be malignant).

a) Benign Tumor: A benign tumor is a tumor is the one that does not expand in an abrupt way; it doesn't affect its neighboring healthy tissues and also does not expand to non-adjacent tissues. Moles are the common example of benign tumors.

b) Pre-Malignant Tumor: Premalignant Tumor is a precancerous phase, considered as a disease, if not correctly preserved it may lead to tumor.

c) Malignant Tumor: Malignancy is the type of tumor that produces worse with the way of stage and initially results in the passing away of a person. Malignant is essentially a medical term that defines a plain regular syndrome. Malignant tumor is a time which is naturally used for the depiction of cancer.

Image Analysis for Brain tumors It deals with unconscious or semi-unconscious methods to support understand the learned images. Due to the large amount of data that is now being caused in the hospitals it is not potential to physically interpret and fragment the data in a practical time. The area of medical image analysis is divided into segmentation and registration, as well as into several further areas like enhancement, visualization, quantification and modeling. Brain tumor studies, segmentation, registration and modeling appear to be the most vital and the most interesting, so the balance of this article emphases on these features.

Segmentation (Pham et al. (2000)) partitions an image into distinct regions containing each pixels with similar attributes. They can be chosen by according to the structures of interst issues types and functional areas. Segmentation is the main step from low-level image processing converting a greyscale or colour image into one or more other images to high-level image depiction in terms of structures, objects, and scenes.

Registration (Maintz and Viergever (1998)). It refers to the determination of one to one communication between the organizes of two or more images such as each consistent point to refer to the same anatomical point. In image registration, the regular conversion maps the locations of an

ISSN: 2250-1177 image to new locations based on the position image. The exact geometrical conversion parameters are the key in the image record-keeping process.(7) The main use of MRIbased medical image analysis for brain tumor studies is in diagnosis, persevering observing and treatment preparation, but it could also be useful in clinical trials. Segmentation is crucial for monitoring of tumor growth or reduction in patients during therapy, for tumor volume measurements and it also plays an vital role in medical preparation or radiotherapy planning, where not only the tumor has to be drawn, but also nearby healthy buildings are of concentration.

Brain imaging techniques Brain imaging methods allow doctors and scientists to view activity or problems within the human brain, without invasive neurosurgery. Number of accepted, safe imaging techniques in use today in research abilities and clinics all over the world. There are many imaging modalities that allow the doctors and researchers to study the brain by looking at the brain non-invasively (Latif, Kazmi, Jaffar, \& Mirza, 2010). MRI provides superior contrast for different brain tissues. It is capable in the request of brain tumor detection and proof of identity, due to the extraordinary gap of soft matters, high spatial resolve and since it does not produce any dangerous radiation, and is a non-intrusive method. MRI offers rich information about mortal soft nerves structure. MRI helps for analysis of the brain cancer. Images obtained by the MRI are used for analyzing and studying the behavior of the brain.(8)Fig1. shows the sample brain MRI.

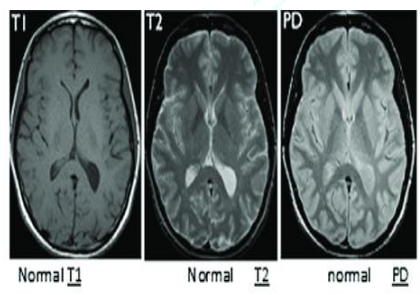

Fig. 1. Normal brain MRI images

The strength of the MRI signal depends primarily on three molecules. Other two parameters are $\mathrm{T} 1$ and $\mathrm{T} 2$ relaxation, which reflect different features of the local environment of individual protons (Latif et al., 2010). The 'pathological' T2 scan is useful for locating the lessened region in the brain. The 'anatomical' T1 scans usually have the best scan resolution, and are useful for localizing anatomical structures. The PD scan displays whole hydrogen thickness per cubic mm.

\section{Methods}

\subsection{Evaluation and Validation Methods}

Validation and comparison against the state of the art is crucial for any newly developed method. Therefore, before diving into the presentation of the di_erent approaches we would like to briey cover the possibilities and challenges for evaluating and validating methods in brain tumor image analysis. It would be optimal to compare any method against the real case. However, this is a big challenge in this _eld, if not impossible. In lack of a well-accepted ground-truth, the current gold standard for evaluation is to compare with manual segmentations by an expert. However, this is an extremely time-consuming and tedious task, additionally it is not objective. Mazzara et al. (2004) reported intra-rater volume variabilities of $20 \%$ _15\% and inter-rater volume variabilities of $28 \%$ _12\% for manual segmentations of brain tumor images. Weltens et al. (2001) found even larger values 
for inter-observer variability. One way to overcome this problem would be to use an algorithm that combines several expert segmentations in an optimal way like STAPLE (War_eld et al. (2004)). This method was applied for the evaluation of brain tumor segmentations by Archip et al. (2007b), however in most cases there are not enough expert segmentations available for using that method. Recently, Xu et al. (2012) suggested to use web-based collaborative manual tumor labeling by a large number of briey trained non-experts to address this problem and they reported encouraging results. A more subjective, but occasionally used way is to employ a semi-automatic segmentation with a different well-accepted method as an intermediate result (e.g. Kikinis and Pieper (2011); Gao et al. (2012); Egger et al. (2013)), which is manually corrected by a human expert where necessary. This approach still lacks in objectivity, but it alleviates the burden from the clinician to spend a large amount of time for segmentation.

Another possibility for a _rest sanity check is to assess results on a synthetic dataset including ground-truth. Although synthetic data lacks important characteristics of real images, it has been used by many groups for initially assessing both segmentation and registration methods on healthy datasets with the BrainWeb phantoms (Cocosco et al.(1997)). For brain tumor studies, Prastawa et al. (2009) made a similar attempt to A Survey of MRI-based Medical Image Analysis for Brain Tumor Studies 7 provide simulated multi-sequence tumor image data including an objective ground truth, which was also based on the BrainWeb phantom and combined with a well-defined and accepted tumor growth model.

The most common way to quantitatively evaluate segmentation or registration results is to calculate the overlap with the ground truth. Usually, Dice similarity coefficient (DSC) or Jaccard coefficients (JC) are used (Crum et al. (2006)). They can range from 0 to 1 with 0 indicating no overlap and 1 indicating perfect overlap. Other possibilities for registration evaluation include manual landmark deffinition and calculation of landmark errors or surface distances. For a more qualitative assessment, checkerboard images can be shown or the outline of a structure can be analyzed visually.

Zou et al. (2004) compared the three different validation metrics: area under the receiver operating characteristics (ROC) curve, mutual information (MI) and Dice similarity coefficient (DSC) for probabilistic brain tumor segmentation. They concluded that for overall classiffcation accuracy the area under the ROC curve should be used, when interested in sensitivity to changes in tumor size MI is the metric of choice and for spatial alignment evaluation the Dice coefficient is best. In the lack of a brain tumor database with ground-truth segmentations, that is available to a broad community of clinicians and researchers, so far most authors validated their algorithms on a limited number of cases from their own data. This makes it difficult to compare the performance of different methods against each other in an unbiased way. Therefore, and due to the different metrics used, the accuracy and speed of the individual methods, which have been collected from the respective publications, can not be directly compared with each other. Until recently, the only data available, which could serve such a purpose of general comparison, was the synthetic data of Prastawa et al. (2009), but so far only few groups tested their methods on these images. For the related topic of MS lesion segmentation from brain MRI (Garc_a-Lorenzo et al. (2013)), an open database has been available for some time (Styner et al. (2008)). Such an open image database has long been missing for an objective comparison of brain tumor segmentation algorithms, however an effort in this direction has _nally been undertaken by the BraTs challenge at MICCAI $2012 \mathrm{x}$.

\subsection{Segmentation of Brain Tumor Images}

Segmentation of tumor-bearing brain images is a challenging task for several reasons. Firstly, high-grade gliomas usually exhibit unclear and irregular boundaries with discontinuities. Contrast uptake and image acquisition time after contrast injection can vary, which changes tumor appearance significantly and it is debatable if and how the non-imageable component of the tumor should be handled by segmentation algorithms. Additionally, tumor subregions can only be separated when several modalities are combined, which requires accurate registration as a preprocessing step. Finally, clinical datasets are usually acquired in a highly anisotropic way, leading to a much higher intra-slice resolution than inter-slice resolution. This causes problems with partial-volume effects for segmentation, but also registration and resampling of the different modalities in a common space of reference.(9) $\mathrm{A}$ diagram illustrating the major steps during the segmentation pipeline of most algorithms is shown infigure 2.

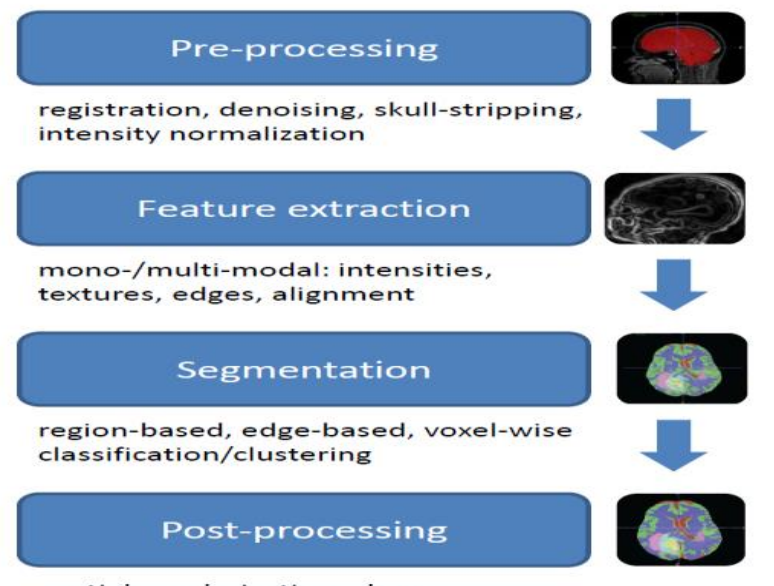

spatial regularization, shape constraints, local constraints

Figure 2. Illustration of the main blocks used for building up the segmentation pipeline of most algorithms included in this review.

\section{Image Pre-processing}

Most algorithms rely on some kind of preprocessing for image preparation and image enhancement. Image denoising is a standard preprocessing task for MRI. Many approaches have been suggested, the most popular ones being based on anisotropic diffusion_altering (Weickert (1998)). Diaz et al. analyzed different denoising algorithms for the specific task of brain tumor segmentation (Diazet al. (2011)). They concluded that, although image noise was reduced, many algorithms introduced artefacts which had a negative effect on segmentation. Intensity normalization (Ny_ul and Udupa (1999)) is a very critical task for MRI, especially when classification methods are used for segmentation. However, for tumor-bearing images, this is more challenging than for healthy images due to the confounding effects caused by the differences in tumor appearance. skin (2011)developed an intensity-normalization technique for MRI, which they claimed was robust A Survey of MRI-based Medical Image Analysis for Brain Tumor Studies 9 to pathologies. Most approaches employ a bias-field correction (Vovk et al. (2007)) before the segmentation is applied, in order to compensate for the e_ect of magnetic feild inhomogeneities 
during image acquisition.When operating on multi-modal images, pre-processing always includes registration of all modalities in a common space of reference. In most cases, this is performed using a linear transformation model with mutual information similarity metric (Mang et al. (2008)) and resampling in order to ensure voxel-to-voxel correspondence across all modalities. Most approaches for brain tumor segmentation rely on a skull-stripping step (Fennema-Notestine et al. (2006)) before the actual segmentation is performed. Speier et al. (2011) recently presented a skull-stripping method dedicated to glioma images, which was able to handle images containing resection cavities(10).

\section{Image Features for Segmentation Algorithms}

The features used for segmentation of brain tumors largely depend on the type of tumor and its grade because different tumor types and grades can vary a lot in appearance (e.g. contrast uptake, shape, regularity, location, etc.). Additionally, feature selection will also depend on the subcompartment of the tumor, which is to be segmented.

The most common feature used for brain tumor segmentation are the image intensities. This is based on the assumption that different tissues have different gray levels. Another type of features frequently used are local image textures because

it has been observed that different tumor areas exhibit di_erent textural patterns (Kassner and Thornhill (2010)). Textures can be computed according to different strategies (Tuceryan and Jain (1998)). Alignment-based features make use of spatial prior knowledge, which is often encoded by registration of a standard atlas to the patient image or by making use of symmetries between left and right brain hemisphere. Intensity gradients or edge-based features can be used for evolving a contour towards the tumor border. Recently, context-aware features modeling mid- or longrange spatial contexts similar to Lepetit et al. (2005) and Shotton et al. (2011) are becoming more popular. Depending on the data available, all these features can either be computed from one single modality or from multi-modal images. Researchers have also combined different features from different modalities in order to improve their segmentation results. For the task of brain tumor image analysis, Schmidt et al. (2005) explored different features for voxel-based classification and concluded that combining textural and alignment-based features allowed for substantial performance increases. Ahmed et al. (2011) investigated the efficacy of different image features and feature fusion strategies for pediatric brain tumor images. They argued that in multi-modal images, texture features had advantages over intensity or shape features.

\section{Segmentation Algorithms}

Based on the previous section, we categorize segmentation algorithms according to the features they use. Therefore, we distinguish region- or edge-based methods, which mostly rely on deformable models.

Classiffication or clustering methods, which make use of voxel-wise intensity and texture features. Many methods employ additional constraints for regularization, this is discussed in a separate section. Another group of methods is based on atlas-based segmentation. Atlas-based methods will be described later in sections 2.3 and 2.4 because the segmentation relies on registration methods(11).

\section{Classiffication and Clustering}

In fact, most of the segmentation algorithms proposed so far, are based on classiffication or clustering approaches. This is mostly owed to the fact that with these methods, multimodal datasets can be handled easily because they can operate on any chosen feature vector. In most cases, the features on which these algorithms A Survey of MRI-based Medical Image Analysis for Brain Tumor Studies 11 operate include voxel-wise intensities and frequently also local textures. The general idea is to decide for every single voxel individually, to which class it belongs based on its feature vector. Classiffication requires training data to learn a classi_cation model, based on which new instances can be labeled. Clustering, on the other hand, works in an unsupervised way and groups data based on certain similarity criteria (Wang and Summers (2012)). Clustering was introduced into the brain tumor segmentation community by Schad et al. (1993) who analyzed texture patterns of different tissues(12). Phillips et al.(1995) employed fuzzy c-means clustering and Vaidyanathan (1995) compared this to kNN-clustering for tumor volume determination during therapy on multispectral 2D image slices. Clark et al. (1998), from the same group, further developed this approach to incorporate knowledge-based techniques. Later, Fletcher-Heath et al.(2001) also combined fuzzy clustering with knowledgebased techniques for brain tumor segmentation from multisequence MRI. Lately, there have been tremendous advances in developing more powerful discriminative classiffication methods and these new methods have also found their way into the yield of medical image analysis. Cai et al. (2007) and later Verma et al. (2008) from the same group, used a high number of MRI modalities (DTI channels in addition to the conventional ones) to create voxel-wise intensity-based feature vectors, which they classi_ed with support vector machines (SVM) (Schoelkopf and Smola (2002)). They were able to not only segment the healthy tissues, but also segment sub-compartments of healthy and tumor regions. Ruan et al. (2007) used a very similar approach based on SVMs, but with a lower number of modalities and they only segmented one tumor region. Later, they claimed that feature selection using kernel class separability, could slightly improve the results compared to their previous approach (Ruan et al. (2011)). Jensen and Schmainda (2009) explored di_erent neural networks to detect brain tumor invasion from multi-parametric MRI (structural, di_usion and perfusion images). Kanaly et al. (2011) chose a simpler approach by thresholding the voxels of the difference image of pre- and post-contrast T1-weighted MRI after intensity normalization. Zikic et al(13). (2012) lately proposed a computationally efficient approach based on decision forest classiffication (Criminisi et al. (2011)) with context-aware features and an additional generative model as an input, which is able to identify tumor sub-compartments from multi-modal images (see _gure 3). They claimed that context-aware features eliminate the need for a postprocessing step imposing smoothness constraints by spatial regularization. Geremia et al. (2012) had the idea to generate synthetic tumor images, which can be used to train a discriminative regression forest algorithm using different groups of features. They argued that this approach allowed them not only to segment patient images, but also to estimate latent tumor cell densities. Characteristics(14) 


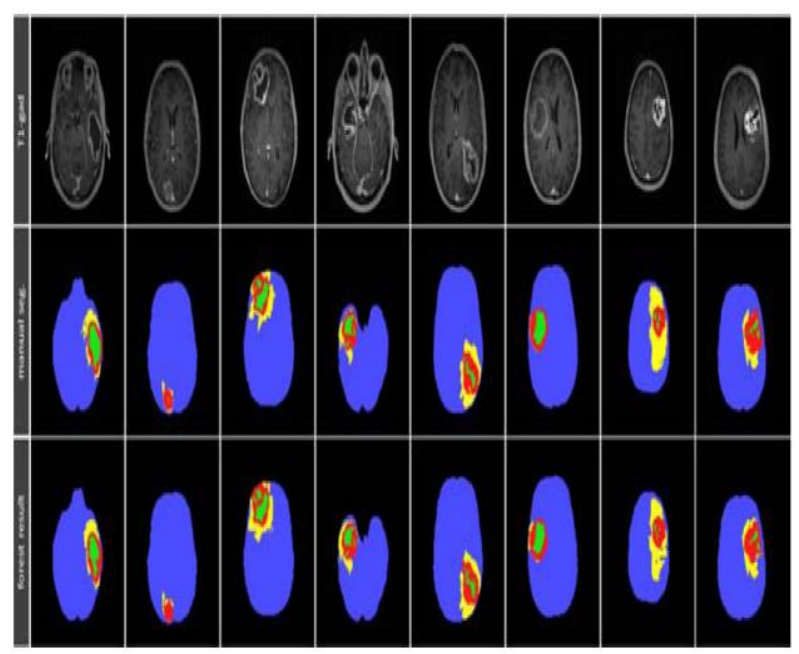

Figure 3. Segmentation results generated from multisequence 3D MR images, shown on T1c-weighted axial slices of different patients. The second row shows the manually defined ground truth, the last row the results of a fully automatic algorithm, which used T1, T1c, T2, T2air and DTI

MRI sequences as an input. The pathologic tissues are separated into active, necrotic and edema compartments. Example from Zikic et al.2012).

\section{References}

[1]. Mohamed Lamine Toure, "Advanced Algorithm for Brain Segmentation using Fuzzy to Localize Cancer and Epilepsy Region", International Conference on Electronics and Information Engineering (ICEIE 2010), Vol. no 2.

[2]. Abdel-Halim Elamy and Maidong Hu (2007) Mining brain tumors \& their growth rates. IEEE Image Processing Society Journal. pp: 872-875.

[3]. T .Logeswari and M.Karnan "An improved implementation of brain tumor detection using segmentation based on soft computing" Journal of Cancer Research and Experimental Oncology Vol. 2(1) pp. 006-014, March, 2010

[4]. Haney SM, Thompson PM, Cloughesy TF, Alger JR and Toga AW (2001) Tracking tumor growth rates in patients with malignant gliomas: a test of two algorithms. AJNR Am. J. Neuroradiology. 22,73-82.

[5].http://dspace.fue.edu.eg/xmlui/bitstream/handle/123456789/ $175 /$ Computer- aided $\% 20$ diagnosis $\% 20$ of $\% 20$ human $\% 20$ brain $\% 20$ tumor $\% 20$ through\%20MRI\%20A\%20survey\%20and\%20a\%20new\%20al gorithm $\% 202014$.pdf?sequence=1\&isAllowed=y

[6].https://boris.unibe.ch/15734/2/BauerPMB2013.pdf

[7].https://www.sciencedirect.com/topics/biochemistry-geneticsand-molecular-biology/image-registration

[8].http://dspace.fue.edu.eg/xmlui/bitstream/handle/123456789/ $175 /$ Computer-

aided $\% 20$ diagnosis $\% 20$ of\%20human $\% 2$ brain $\% 20$ tumor $\% 20$ through\%20MRI\%20A\%20survey\%20and\%20a\%20new\%20al gorithm $\% 202014$.pdf?sequence=1\&isAllowed=y

[9]. Ahmed, S., Iftekharuddin, K. M., and Vossough, A. (2011). E_cacy of texture, shape,and intensity feature fusion for posterior-fossa tumor segmentation in MRI. IEEEtransactions on information technology in biomedicine : a publication of the IEEEEngineering in Medicine and Biology Society, 15(2):206\{13.

[10]. Ambrosini, R. D., Wang, P., and O'Dell, W. G. (2010). Computeraided detection of metastatic brain tumors using automated three-dimensional template matching. Journal of magnetic resonance imaging : JMRI, 31(1):85\{93.

[11].Andersen, S. M., Rapcsak, S. Z., and Beeson, P. M. (2010). Cost function masking during normalization of brains with focal lesions: still a necessity? NeuroImage, 53(1):78\{84.

[12].Angelini, E. D., Clatz, O., Mandonnet, E., Konukoglu, E., Capelle, L., and Du_au, H. (2007). Glioma Dynamics and Computational Models: A Review of Segmentation, Registration, and In Silico Growth Algorithms and their Clinical Applications. Current Medical Imaging Reviews, 3(4):262\{276

[13].Angelini, E. D., Delon, J., Bah, A. B., Capelle, L., and Mandonnet, E. (2012).Di_erential MRI analysis for quanti_cation of low grade glioma growth. Medical image analysis, 16(1):114\{26.

[14].Archip, N., Clatz, O., Whalen, S., Kacher, D., Fedorov, A., Kot, A., Chrisochoides, N.,Jolesz, F., Golby, A., Black, P. M., and War_eld, S. K. (2007a). Non-rigid alignment of pre-operative MRI, fMRI, and DT-MRI with intra-operative MRI for enhanced visualization and navigation in image-guided neurosurgery. NeuroImage, 35(2):609\{24.

[15]. Archip, N., Jolesz, F. A., and War_eld, S. K. (2007b). A validation framework for brain tumor segmentation. Academic radiology, [14].(10):1242\{51. Bach Cuadra, M., De Craene, M., Duay, V., Macq, B., Pollo, C., and Thiran,J.-P. (2006). Dense deformation _eldestimation for atlas-based segmentation of pathological MR brain images. Computer methods and programs in biomedicine,84(2-3):66\{75. 